\title{
Una aproximación a la implementación de los estudios de futuro en el Servicio Geológico Colombiano
}

\author{
An approach to the implementation of future studies in the \\ Colombian Geological Survey
}

Guillermo Parrado Lozano ${ }^{1}$

Recibido: 21 de agosto de 2019

Aceptado: 11 de diciembre de 2019

Publicado en línea: 30 de diciembre de 2019

Doi: https://doi.org/10.32685/2590-

7468/invapnuclear.3.2019.512

Palabras clave: estudios de futuro, prospectiva, geociencias, ciencias nucleares, centro de pensamiento.

Keywords: future studies, foresight, geosciences, nuclear sciences, think $\operatorname{tank}$
Citación: G. Parrado Lozano, "Una aproximación a la implementación de los estudios de futuro en el Servicio Geológico Colombiano”, Revista Investigaciones y Aplicaciones Nucleares, $\mathrm{n}{ }^{\circ}$ 3, pp. 49-68, 2019. https://doi.org/10.32685/2590-7468/invapnuclear.3.2019.512

\section{RESUMEN}

Se presenta una revisión bibliográfica relacionada con los conceptos fundamentales y metodologías aplicables en una estrategia de construcción social del conocimiento de los escenarios posibles, probables y deseables para el desarrollo del Servicio Geológico Colombiano —entidad pública nacional de ciencia y tecnología - en el siglo XXI. Partiendo de los conceptos fundamentales, luego se muestra una breve reseña histórica de los desarrollos de la prospectiva como disciplina, para finalizar con una aproximación al rol de las geociencias y las ciencias nucleares, pilares de la consolidación del Servicio Geológico Colombiano (SGC) como líder en el ámbito de sus competencias en nuestro país.

\section{ABSTRACT}

A bibliographic review is presented of fundamental concepts and methods applicable in a social construction of knowledge strategy for possible, probable and desirable scenarios for the development of the Colombian Geological Survey, a national science and technology public entity, in the twenty-first century. Starting from fundamental concepts, a brief historical review of the development of foresight as a discipline is provided, ending with an approach to the role of the geosciences and nuclear sciences, pillars of the consolidation of the Colombian Geological Survey as a leader in the field of its competencies in Colombia.

\footnotetext{
${ }^{1}$ Dirección de Asuntos Nucleares, Servicio Geológico Colombiano. Coordinador del Grupo de Reactor Nuclear de Investigación IAN-R1. Líder del Grupo de Investigación Asuntos Nucleares, de Minciencias.

Email de correspondencia: gparrado@sgc.gov.co
} 


\section{MARCO CONCEPTUAL}

$\mathbf{L}$ a predicción, el pronóstico o anticipación del futuro ha sido uno de los retos más inquietantes en el tiempo de cada una de las antiguas civilizaciones, y ahora, en tiempos posmodernos, sigue vigente. La necesidad de saber con antelación cómo sería el clima para sembrar y cosechar con éxito, el conocimiento estimado de la calidad, cantidad y duración de las reservas de agua o alimentos y su eventual racionamiento en épocas de escasez, así como el crecimiento de la población que haría uso de estos, son apenas algunos de los interrogantes a los que se veían enfrentados de forma cotidiana nuestros más primitivos antecesores.

Situados en el terreno bélico, los antiguos empezaron a desarrollar capacidades a priori de modelación del futuro para conocer la composición, disposición y posibilidades de movilización o ataque de los ejércitos de sus adversarios. El arte de la anticipación también aparece ligado al arte de la guerra. El maestro Sun Tzu, en su texto clásico El arte de la guerra, indica que

Si intentas utilizar los métodos de un gobierno civil para dirigir una operación militar, la operación será confusa. Triunfan aquellos que saben cuándo luchar y cuándo no. Saben discernir cuándo utilizar muchas o pocas tropas. Tienen tropas cuyos rangos superiores e inferiores tienen el mismo objetivo. Se enfrentan con preparativos a enemigos desprevenidos. Tienen generales competentes y no limitados por sus gobiernos civiles. Estas cinco son las maneras de conocer al futuro vencedor. [1]

En la literatura de anticipación, también conocida como relatos de ciencia ficción, se ofrece otra interesante aproximación a escenarios futuros, prometedores o demoledores. Autores de todas las épocas y matices, como Agustín de Hipona, Leonardo da Vinci, Tomás Moro, Francis Bacon, Charles Fourier, Julio Verne, Ray Bradbury, Isaac Asímov, H. G. Wells, Aldous Huxley, George Orwell, Katshuiro Ōtomo y Tom King, por nombrar solo a algunos de ellos, han contribuido desde su particular genialidad en su respectivo campo, a la previsión de las posibles consecuencias de la introducción de determinados avances tecnológicos en imaginarias sociedades normalizadas o distópicas [2].

Los denominados estudios de futuro (foresight, en su denominación anglosajona), citados por la Sociedad Finlandesa de Estudios del Futuro, se definen como "los estudios [que] examinan el presente con una especial comprensión del futuro, integran resultados de investigación de diferentes campos de conocimiento y ayudan a los encargados de la adopción de decisiones estratégicas a hacer mejores elecciones para un futuro común". Lo cual significa que conceptualmente estos se enriquecen de los aportes multidisciplinares y se caracterizan porque ven "el futuro como un espacio de realización del potencial humano, donde caben múltiples alternativas que permiten pensar y gestionar, bajo una óptica del bien común, la incertidumbre asociada a las decisiones estratégicas que marcan la trayectoria de la sociedad en su conjunto" [3].

En la tabla 1 se exhibe una descripción de la estructura los diferentes futuros, se muestra su denominación de uso común o informal y la denominación técnica, según el investigador prospectivista colombiano Carlos Mera [4].

Tabla 1. Estructura del futuro

\begin{tabular}{cc}
\hline $\begin{array}{c}\text { Discurso } \\
\text { teórico-práctico }\end{array}$ & Designación aceptada \\
\hline Adivinar & Adivinación \\
\hline Predecir & Profecía \\
\hline Anticipar & Futurología \\
\hline Construir el futuro & Prospectiva \\
\hline Imaginar & Ciencia ficción \\
\hline Soñar & Utopía \\
\hline
\end{tabular}

Los estudios de futuro, en la particular visión de Mera, presuponen un papel activo de los seres humanos, en cuanto se orientan a su involucramiento en la modificación de las posibles realidades en las que desarrollarán su existencia. Este involucramiento determina la voluntad y necesidad de cambio de un presente que muchas veces se puede mostrar desgastado, agotado, cíclico o repetido, es decir, desprovisto de esperanzas, retos o desafíos que pongan a prueba nuestras capacidades intelectuales.

En la figura 1 se muestra el escenario convergente de aparición de la prospectiva en la década de los noventa, resultado de los enfoques de la planificación estratégica, los es- 
tudios de futuro y el conexo análisis de las políticas públicas. Gavigan (citado en [4]) asegura que

La prospectiva ha tenido tendencia a ser más frecuente en aquellos campos socioeconómicos en los que se puede partir de una base de indicadores y de datos, y en las que las tendencias y contratendencias establecidas (o al menos ampliamente percibidas) son la base de las inversiones a largo plazo, como en las áreas de la ciencia, la tecnología y la innovación.

Los cuatro sentidos básicos de la prospectiva se describen a continuación [3]:

» Pensar en el futuro (anticipación): Los estudios más clásicos en materia de anticipación son los que tienen que ver con pensar el futuro. Esto significa, entre otras cosas, plantear imágenes del futuro, diseñar escenarios, anunciar alertas tempranas e identificar tendencias.

» Debatir sobre el futuro (apropiación): Debatir sobre el futuro implica dar un paso más adelante y plantear nuevas ideas que enriquezcan un diálogo con toda la sociedad, teniendo en cuenta los resultados del estudio de escenarios futuros. En este caso se trata de incluir nuevos temas en la agenda pública, lo que pone de relieve el vínculo entre prospectiva e innovación. Estos nuevos temas tienen que ver con múltiples dimensiones de la realidad, bien sea al nivel político, económico, social, ambiental, de ciencia y tecnología, de participación ciudadana o de seguridad.

» Modelar el futuro (acción): Modelar o dar forma al futuro tiene que ver sobre todo con preparar procesos de decisión sistemáticos y organizados, que entrañan acumulaciones de conocimiento, resultados progresivos, generación de capacidades que preparan a la sociedad para la toma de decisiones acerca de su futuro, de una manera coherente y durante largos períodos de tiempo.

» Vigilar el presente (aprendizaje): La vigilancia estratégica del presente está íntimamente relacionada con metodologías afines con el análisis de riesgo y el análisis de horizontes o análisis del entorno. Gestiona los sistemas participativos y basados en datos conceptuales, innovaciones metodológicas, tecnológicas y de comunicación, capaces de apoyar la identificación, evaluación y explotación de conocimientos relacionados con cuestiones complejas y altamente inciertas, como las sorpresas, las "cartas salvajes" (wild cards) y las señales débiles, así como los asuntos emergentes que se derivan de la interconexión de conocimientos provenientes de comunidades de exploración e investigación al nivel mundial.

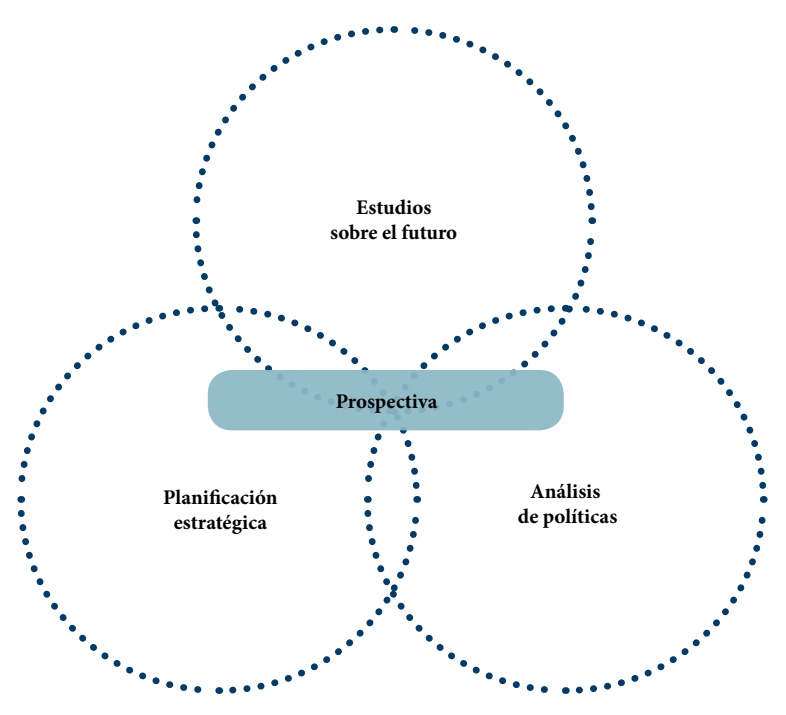

Figura 1. Enfoque procedente de la prospectiva Fuente: [4]

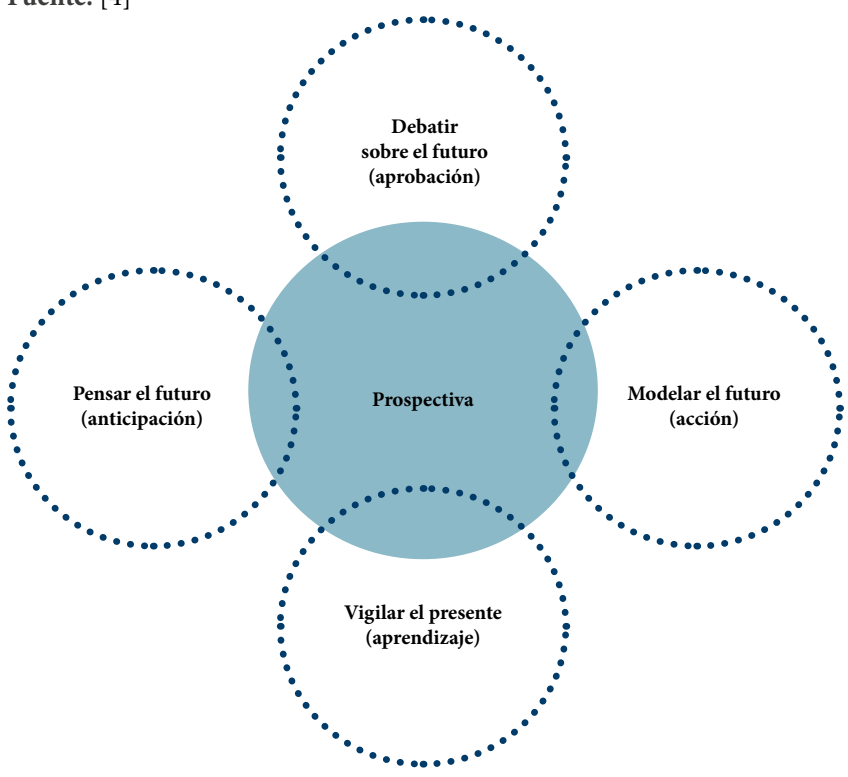

Figura 2. Sentidos básicos de la prospectiva [4]

Los estudios de futuro también suelen relacionarse con cuatro categorías conceptuales básicas que se describen en la tabla 2. 
Tabla 2. Conceptos básicos de los estudios de futuro (adaptado de [4]

\begin{tabular}{|c|c|c|}
\hline Nivel & Definición & Pertinencia \\
\hline 1. Predicción & $\begin{array}{l}\text { Una declaración no probabilística, con un nivel de confianza absoluto } \\
\text { acerca del futuro. Por "no probabilística" se entiende que es un enunciado } \\
\text { que tiene la pretensión de ser único, exacto y no sujeto a controversia. }\end{array}$ & $\begin{array}{l}\text { Pertinente en las ciencias exactas, en las que existen leyes, } \\
\text { verificación experimental y control de variables. }\end{array}$ \\
\hline 2. Pronóstico & $\begin{array}{l}\text { Pretende determinar la probabilidad de que ocurran ciertos eventos } \\
\text { futuros, con un nivel de confianza relativamente alto. Se centra en la } \\
\text { calidad de los enunciados y las interpretaciones realizadas. }\end{array}$ & $\begin{array}{l}\text { En materia de ciencias sociales, ciencias de la administración y } \\
\text { estudios del futuro, es más pertinente hablar de pronósticos que de } \\
\text { predicciones. }\end{array}$ \\
\hline 3. Prospectiva & $\begin{array}{l}\text { Se exploran diferentes tipos de incertidumbre: } \\
\text { » la incertidumbre suave se presenta cuando la dinámica del fenómeno } \\
\text { sigue alguna forma ordenada, aun si es compleja, como las cadenas } \\
\text { estocásticas o las oscilaciones de largo plazo, y } \\
\text { » la incertidumbre dura es inherente a la estructura interna de la } \\
\text { dinámica del fenómeno, que se comporta, al menos parcialmente, de } \\
\text { un modo caótico, indeterminado y casual desde la perspectiva actual } \\
\text { del pensamiento humano. }\end{array}$ & $\begin{array}{l}\text { La incertidumbre se origina en la deficiencia de los métodos o } \\
\text { en la inadecuada comprensión del proceso y no en la estructura } \\
\text { misma del fenómeno. } \\
\text { „ La incertidumbre dura incluye las denominadas situaciones } \\
\text { explosivas, las tendencias declinantes y las situaciones propensas } \\
\text { a sorpresas. }\end{array}$ \\
\hline 4. Pensamiento complejo & $\begin{array}{l}\text { Aborda la complejidad que surge cuando el entorno se torna opaco y } \\
\text { menos descifrable, debido a que el contexto se modifica constantemente } \\
\text { por el efecto de las acciones de los actores internos y externos. }\end{array}$ & $\begin{array}{l}\text { Explora la ambigüedad que afecta la capacidad de operar de las } \\
\text { organizaciones. }\end{array}$ \\
\hline
\end{tabular}

Para Mario Bunge, el conocido físico y filósofo de la ciencia argentino, es necesario hacer una primera distinción conceptual entre la prospectiva y la predicción. Cabe aclarar que este autor utiliza también los términos prognosis, conjetura y expectativa, entre otros [5]. En la tabla anterior Medina desarrolla de manera creciente estas categorías enfatizando en su definición y en la pertinencia de aplicarlas, partiendo de la simple predicción hasta llegar al pensamiento complejo, categoría ampliamente estudiada por Edgar Morin, en una nueva lógica denominada complejidad, definida como "un fenómeno cuantitativo, una cantidad extrema de interacciones e interferencias entre un número muy grande de unidades". ¿Qué es la complejidad, entonces?

Según Morin [6],

A primera vista la complejidad es un tejido (complexus: lo que está tejido en conjunto) de constituyentes heterogéneos inseparablemente asociados: presenta la paradoja de lo uno y lo múltiple. Al mirar con más atención, la complejidad es, efectivamente, el tejido de eventos, acciones, interacciones, retroacciones, determinaciones, azares, que constituyen nuestro mundo fenoménico. Así es que la complejidad se presenta con los rasgos inquietantes de lo enredado, de lo inextricable, del desorden, de la ambigüedad, la incertidumbre [...] De allí la necesidad, para el conocimiento, de poner orden en los fenómenos rechazando el desorden, de descartar lo incierto, es decir, de seleccionar los elementos de orden y de certidumbre, de quitar ambigüedad, clarificar, distinguir, jerarquizar [...] Pero tales operaciones, necesarias para la inteligibilidad, corren el riesgo de producir ceguera si eliminan a los otros caracteres de lo complejo; $y$, efectivamente, como ya se ha indicado, nos han vuelto ciegos [...] Finalmente, se hizo evidente que la vida no es una sustancia, sino un fenómeno de auto-eco-organización extraordinariamente complejo que produce la autonomía. Desde entonces es evidente que los fenómenos antropo-sociales no podrían obedecer a principios de inteligibilidad menos complejos que aquellos requeridos para los fenómenos naturales

Esta ceguera tiene que ver con la entronización de la simplificación, de la visión reduccionista, del anhelo profundamente humano de encontrar teorías generales que expliquen de forma unidimensional toda la multidimensionalidad de los cambios, cada vez más acelerados, que vivimos en la sociedad actual [7].

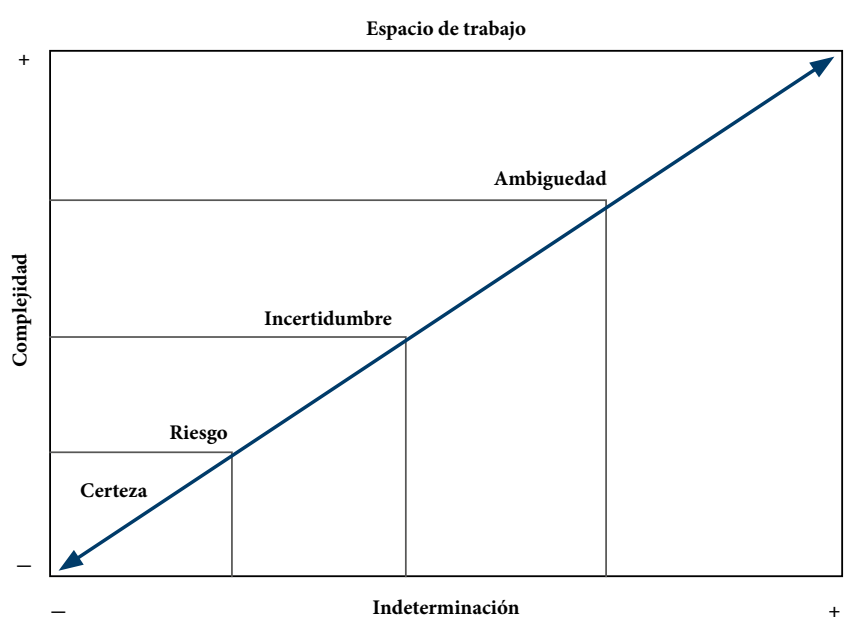

Figura 3. Relaciones entre la complejidad y la indeterminación (adaptado de [4] 
Tabla 3. Relaciones entre los niveles de indeterminación y los conceptos básicos

\begin{tabular}{|c|c|c|c|c|c|}
\hline Nivel & $\begin{array}{l}\text { Orientación hacia el } \\
\text { futuro }\end{array}$ & $\begin{array}{l}\text { Predictibilidad de los } \\
\text { eventos }\end{array}$ & Situaciones futuras & Tipo de futuro y estrategia & Métodos \\
\hline 1. Certeza & Predicción & $\begin{array}{l}\text { Eventos que ciertamente } \\
\text { ocurrirán }\end{array}$ & Deterministas & $\begin{array}{l}\text { Futuro suficientemente } \\
\text { claro. Es viable cierto nivel } \\
\text { de predicción }\end{array}$ & $\begin{array}{l}\text { Análisis y monitoreo del } \\
\text { entorno }\end{array}$ \\
\hline 2. Riesgo & Pronóstico & $\begin{array}{l}\text { Eventos que } \\
\text { probablemente ocurrirán }\end{array}$ & $\begin{array}{l}\text { Aproximables por } \\
\text { hipótesis convencionales } \\
\text { de regularidad } \\
\text { estocástica. Se establecen } \\
\text { probabilidades de que } \\
\text { ocurran ciertos eventos, } \\
\text { de acuerdo con unos } \\
\text { argumentos determinados }\end{array}$ & $\begin{array}{l}\text { Futuros alternativos. } \\
\text { Se sabe cuáles son los } \\
\text { actores, las variables y } \\
\text { las interrelaciones que } \\
\text { se manejan, y se pueden } \\
\text { plantear sus probables } \\
\text { futuros }\end{array}$ & $\begin{array}{l}\text { Análisis de tendencias y } \\
\text { escenarios cuantitativos }\end{array}$ \\
\hline 3. Incertidumbre & Prospectiva & $\begin{array}{l}\text { Universo conocido de los } \\
\text { estados posibles. Eventos } \\
\text { que podrían ocurrir } \\
\text { dentro de un espectro de } \\
\text { situaciones conocidas, sin } \\
\text { que se sepa la secuencia } \\
\text { en que se producirían }\end{array}$ & $\begin{array}{l}\text { Preidentificadas, aunque } \\
\text { se desconozca la cadena } \\
\text { de eventos que las pueda } \\
\text { producir. Es difícil } \\
\text { conocer a todos los } \\
\text { actores, las variables y } \\
\text { las interrelaciones que se } \\
\text { manejan }\end{array}$ & $\begin{array}{l}\text { Se plantean futuros } \\
\text { posibles. Una gama de } \\
\text { posibles resultados, pero } \\
\text { sin escenario natural } \\
\text { alguno. No se conoce la } \\
\text { probabilidad de ocurrencia } \\
\text { de un evento }\end{array}$ & $\begin{array}{l}\text { Consulta de expertos, } \\
\text { escenarios cualitativos }\end{array}$ \\
\hline 4. Ambigüedad & Pensamiento complejo & $\begin{array}{l}\text { Eventos francamente } \\
\text { indeterminados. } \\
\text { Incertidumbre no } \\
\text { estructurada. }\end{array}$ & $\begin{array}{l}\text { Acciones y reacciones } \\
\text { caracterizadas por la } \\
\text { creatividad, la innovación } \\
\text { y la sorpresa }\end{array}$ & $\begin{array}{l}\text { Auténtica ambigüedad. Sin } \\
\text { base alguna para predecir el } \\
\text { futuro, ya que no se pueden } \\
\text { identificar, y menos prever, } \\
\text { las variables pertinentes } \\
\text { para configurar el futuro. } \\
\text { Ni siquiera sería posible } \\
\text { elaborar escenarios para el } \\
\text { diseño de una estrategia }\end{array}$ & $\begin{array}{l}\text { Simulación y modelación } \\
\text { no lineal. Creatividad }\end{array}$ \\
\hline
\end{tabular}

Fuente: adaptada de [4]

La complejidad, entendida como el espacio de la no-linealidad, de las múltiples, variadas y simultáneas interacciones, se contrapone a las teorías de elección racional y presupone que nuestras decisiones están lejos de la precitada visión reduccionista. Como se muestra en la figura 3 , se postula un amplio espectro del conocimiento, que comprende cuatro estadios básicos, que van desde la certeza hasta la ambigüedad, pasando por el riesgo y la incertidumbre. Esos estadios, representados en un plano cartesiano, en uno de sus ejes muestra la relación entre el determinismo y la indeterminación, y en el otro, la relación entre la baja y la alta complejidad de los espacios de trabajo de la prospectiva. En la certeza se conocen de forma evidente las variables y sus relaciones. Se presenta el riesgo cuando se conocen las variables, pero tan solo se pueden estimar las relaciones existentes entre ellas, o sea, sus probabilidades. Se observa la incertidumbre si se conocen las variables, pero algunas no se pueden medir, y se desconocen las relaciones entre todas. Finalmente, la ambigüedad se presenta si no se conocen todas las variables pertinentes y estas son sujetos de identificación [8].

Las referidas relaciones entre la complejidad y la determinación dan cuenta de la vasta aplicabilidad de los estudios prospectivos en temas tan variados como los de defensa, seguridades nacional, energética y alimentaria, modelos de negocios, análisis de mercados, análisis electoral, megatendencias en ciencias, modelos de predicción del clima, entre otros. Este escenario se enmarca en los postulados de Michel Godet, uno de los notables teóricos de esta disciplina, quien indica que por fortuna la única certidumbre es la muerte, pues un presente caracterizado por la certeza y la predictibilidad sería, en últimas, insoportable. Esta afirmación la sustenta en la premisa relativa a que el futuro no es la prolongación determinista del pasado, sino que este está abierto a la conjunción de múltiples actores que se mueven en función de los interese de sus proyectos, donde, en todo caso, la idea-fuerza es que "la anticipación es necesaria para iluminar la acción” [9].

Otro importante autor de la transdisciplina prospectiva es Bertrand de Jouvenel (citado en [4]), uno de sus pioneros mundiales, quién anota que

Al observar el pasado, la voluntad del hombre es vana, su libertad es nula, su poder, inexistente [...] El pasado es el lugar de los hechos sobre los que no se puede hacer nada, es al mismo tiempo el lugar de los hechos conocidos y constatados. Mientras que, por el contrario, el futuro es 
para el hombre, en tanto que sujeto dotado de conocimiento y razón, espacio de incertidumbre, y en tanto que sujeto activo, espacio de libertad y de poder.

Sin embargo, son varios los autores que sitúan a Gastón Berger (1957) como el creador del vocablo prospectiva, que, en oposición al término retrospectiva, se define como "la ciencia que estudia el futuro para poder influir en él". Ben Martin (citado en [4]) describe la prospectiva como "El proceso de investigación que requiere mirar sistemáticamente el futuro a largo plazo en ciencia, tecnología, economía y sociedad, con el objetivo de identificar las áreas de investigación estratégicas y las tecnologías genéricas emergentes que generarán los mayores beneficios económicos y sociales".

Reconstruyendo los antecedentes más remotos de los estudios del futuro, los investigadores Ortegón y Medina [10] indican que ya Platón, en su República, describía “una sociedad futura donde el concepto de justicia es capital para la vida e instituciones sociales”. También mencionan cómo san Agustín, obispo de Hipona, en su obra La ciudad de Dios, se sitúa en una ciudad terrena asociada a la ambición humana, y su contrapuesta ciudad divina, fundamentada en el amor de Dios, cuya configuración solo puede ser posible en la tierra cuando sus estructuras sociales, en el futuro, se alinearen con la voluntad del Creador. En la Utopía de Tomás Moro, este pensador inglés describe una sociedad idealizada en la que los individuos conviven de forma armónica en la entidad construida, que se fundamenta en el bien común, la educación y el trabajo como sus pilares. Francis Bacon, otro filósofo inglés, relaciona La nueva Atlantis como como ese espacio de futuro prometedor donde el individuo fundamenta su poder y su autonomía. Ya a finales del siglo XIX, los pensadores Auguste Comte y Karl Marx, de manera coincidente, postularon que la ciencia y la tecnología, como motores del progreso y el cambio social, coadyuvarían en la solución de muchos de los problemas que aquejaban a las sociedades de ese entonces (salubridad, movilidad, pobreza, inequidad, entre otros), premisa que aún tiene vigencia hoy en día [10].
Teniendo en cuenta que los estudios del futuro en efecto están revestidos de un carácter científico sin un objeto definido, es posible llegar a la comprensión de tres tipos de futuro: posible, probable y deseable, cuya descripción se muestra en la tabla 4 [9].

En este orden de ideas, los estudios de futuro se rigen -en un consenso ya reconocido a finales del siglo XX - por tres principios fundamentales, que se describen a continuación [9]:

\Primer principio. Reconoce la existencia de un dilema característico de los estudios de futuro entre el conocimiento y el deseo/temor. Mientras que necesitamos conocer el pasado y el presente como referentes para analizar el futuro, por otro lado nuestros deseos/temores acerca de este último no se corresponden con nuestros conocimientos, y hasta eventualmente los contradicen.

\Segundo principio. El único tiempo sobre el cual los humanos podemos tener incidencia es el futuro. El pasado es el tiempo de los hechos cumplidos. El presente es este momento que ahora vivimos, que en unos instantes ya será pasado. Antonio Concheiro, notable teórico de la disciplina, establece que “...el pasado pertenece a la memoria, el presente a la acción, y el futuro, a la imaginación y la voluntad...”.

» Tercer principio. Postula que no hay un solo futuro, sino muchos futuros posibles. Aquí bien vale mencionar el significado del término futuribles, esbozado por De Jouvenel (citado por Mera) como "el complejo de futuros alternativos posibles y probables mostrados como construcciones mentales" [4].

En un reciente estudio - pleno de didáctica-, Hauptman y Steinmüller indagan acerca de las definiciones y diferencias entre los relatos de anticipación o de ciencia ficción y los estudios de futuro. Partiendo de la premisa ¿Qué pasaría si...? exploran una interesante senda que integra de manera metódica la imaginación en el proceso de construcción social de futuros. En la tabla 5 se muestran las principales variables que describen estas dos aproximaciones [39].

\begin{tabular}{ll}
\hline Los futuros posibles & Exploran alternativas mutables, sujetas a incertidumbres y rupturas o discontinuidades. \\
\hline Los futuros probables o esperados & Son resultado del análisis de tendencias y de extrapolaciones de "datos" del presente. \\
\hline Los futuros deseables o preferidos & $\begin{array}{l}\text { Reflejan la expectativa de atención de las demandas actuales de la sociedad, de políticas de gobierno, de estrategias empresariales, } \\
\text { entre otras, que se expresan por medio de las metas o los valores de los actores sociales. }\end{array}$ \\
\hline
\end{tabular}


Tabla 5. Variables descriptivas de la ciencia ficción y de los estudios de futuro

\begin{tabular}{|c|c|c|}
\hline & Ciencia ficción & Prospectiva \\
\hline Objetivo & Entretenimiento. Estimulación intelectual. & Proporcionar orientación para la acción. \\
\hline Enfoque & Intuitivo, creativo (con métodos artísticos de ficción). & $\begin{array}{l}\text { Acorde con lo científico / mejores prácticas } \\
\text { metodológicas / creatividad. }\end{array}$ \\
\hline Cuestiones orientadoras & $\begin{array}{c}\text { ¿Qué es imaginable? } \\
\text { ¿Cuáles son las implicaciones más sorprendentes, } \\
\text { increíbles y desastrosas? }\end{array}$ & $\begin{array}{c}\text { ¿Qué es posible? } \\
\text { ¿Qué es probable? } \\
\text { ¿Qué es deseable? } \\
\text { ¿Qué implicaciones son admisibles? } \\
\end{array}$ \\
\hline Retos & $\begin{array}{l}\text { Suspensión de la incredulidad. } \\
\text { Inducción de una sensación de asombro. }\end{array}$ & Nuevas ideas convincentes y útiles sobre el o los futuros. \\
\hline Criterios de calidad & $\begin{array}{l}\text { Originalidad. Visiones poderosas y convincentes. } \\
\text { Estilo y calidades dramáticas. Personajes convincentes. }\end{array}$ & $\begin{array}{c}\text { Plausibilidad, coherencia lógica. } \\
\text { Realismo. } \\
\text { Transparencia metodológica (con respecto a las } \\
\text { declaraciones de valores). }\end{array}$ \\
\hline Factores de éxito & Placer/satisfacción del lector. Ventas. & $\begin{array}{c}\text { Satisfacción de los clientes. } \\
\text { Utilidad para tomar las mejores decisiones. }\end{array}$ \\
\hline
\end{tabular}

Fuente: [38]

Estos investigadores finalmente señalan que la previsión genera imágenes del futuro que pueden ser asimiladas con métodos controlados, fundamentados en el mejor conocimiento disponible de los dominios de lo posible, de manera que la creatividad y la imaginación son insumos necesarios por varias razones: para superar la miopía de "presentismo", también para integrar los aspectos humanos ("humanidad") en la imagen del futuro $y$, finalmente, para traer al presente algunas de las muy variadas posibilidades en las que, por supuesto, el futuro es bastante pródigo.

\section{APUNTES DE PROSPECTIVA EN GEOCIENCIAS Y EN CIENCIAS NUCLEARES}

La prospectiva, como otras disciplinas de su naturaleza, tiene ahora, en su madurez, un cuerpo conceptual y metodológico robusto, que le imprime solidez, identifica sus ámbitos de competencia y establece sus dominios temáticos, que se avizoran en continua expansión. Un objetivo de este artículo es mostrar sucintamente algunas de las cuestiones de relevancia actual en las disciplinas relacionadas, que conciten el interés de los investigadores y de los que sienten curiosidad por estas temáticas. En primera instancia se muestran algunos desarrollos conceptuales del campo geocientífico, donde el elemento articulador es de naturaleza antropocéntrica, es decir, orientado hacia la satisfacción de las necesidades de la especia humana. Luego se muestra lo pertinente a las ciencias nucleares, haciendo hincapié en un campo particular, el de la seguridad energética, que cobra una especial importancia en el actual momento histórico, cuando se exponen - algunas veces sin la debida profundidad y rigor- argumentos a favor o en contra de la diversi- ficación de la canasta energética, el agotamiento de las fuentes tradicionales de suministro de materias primas y la posibilidad de armonizar el consumo con la sostenibilidad planetaria.

\subsection{Prospectiva en geociencias}

Los rasgos interdisciplinarios se revelan en aproximaciones tan disímiles como las de las geociencias y el arte. En un reciente número del Journal of Maps [11], el editorial destaca cómo las colaboraciones entre artes y geociencias ayudan a comprender el levantamiento de información espacial en geomorfología, cartografía geológica, estudios del Cuaternario, etc., a partir de la elaboración de mapas cuya representación gráfica se fundamenta en tres conceptos críticos: espaciales (distancias, direcciones, etc.), locales (territorios, delimitaciones, etc.) y ambientales (características biofísicas, etc.), donde los humanos y nuestro dinamismo (pasado, presente y futuro) predeterminamos en buena medida la construcción de los sitios donde habitamos o proyectamos hacerlo. Los mapas, como representaciones de esas posibles realidades, pueden acudir al arte, en un interesante ejercicio que deja atrás el viejo paradigma que evidenciaba la desconexión entre las humanidades y las ciencias [11].

En este orden de ideas, entre los múltiples ámbitos de estudio conocidos en prospectiva, se menciona la emergencia del término prospectiva territorial, asimilado a aquello que en el ámbito latinoamericano el investigador mexicano Arturo Montañana (citado por Baena) denominó un "modelo propio de la geoprospectiva", postulado como una teoría orientada a la elaboración de "herramientas que permitan construir los futuros alternativos, geográficamente situados, que sirvan a las comunidades, individuos o instituciones, 
para mejorar la calidad de vida de sus miembros, alcanzar la sustentabilidad de sus lugares, y fortalecer su cohesión social" [12]. La prospectiva territorial es una disciplina que incluye los siguientes modelos referidos al concepto integral del ordenamiento territorial:

Modelo para determinar las tendencias clave en unidades de paisaje y del territorio (según sus características o propiedades)

\) Modelo de órdenes clasificatorios de tendencias

ı Modelo para determinar los escenarios probables, posibles y deseables

\) Modelo para determinar el peso de los actores territoriales

\) Modelo para el pronóstico y análisis de configuraciones territoriales

\) Modelo para analizar acciones y determinar el programa político relacionado con su ejecución

Este modelo y su aplicación práctica contribuyen a visualizar los posibles escenarios de desarrollo de las comunidades, como lo menciona su autor, en búsqueda de la armonía entre estas y su entorno natural [12].

La misma interdisciplinariedad mencionada al principio de este aparte se constituye en un elemento central del estudio acerca de la proyección del futuro de las investigaciones en hidrogeología [13]. La hidrogeología, como otros campos del saber, no ha sido ajena a las dinámicas de cambio caracterizadas por los conflictos entre la adopción de los enfoques reduccionistas y los alternativos interdisciplinarios. De esta forma, desde mediados del siglo XX, y hasta la primera década del XXI, la cuantificación y el modelamiento de los recursos hídricos fueron los objetos centrales de estudio e investigación en esta disciplina. Otros temas que se prevé podrán abordarse en décadas posteriores se relacionan con la sostenibilidad del recurso (ecología subterránea, simulaciones demanda-suministro, interacciones biogeoquímicas, etc.), los estudios de complejidad (aproximaciones estocásticas, determinísticas, teoría del caos, etc.), las aproximaciones reduccionistas rigurosas (repetibilidad, generalización, etc.), la heterogeneidad (porosidad primaria y secundaria, secuencias estratigráficas, modelos geoestadísticos, etc.), la incertidumbre (demanda del recurso, salud pública, vacíos de información, etc.) y, finalmente, las proyecciones en in-

vestigación básica y aplicada asociadas al estudio de los geosistemas complejos no lineales [13].

En cuanto a la proyección de las geociencias y sus ámbitos de estudio, el Committee on Grand Research Questions in the Solid-Earth Sciences, con el auspicio de las Academias Nacionales de Ciencias, Ingeniería y Medicina de los Estados Unidos, formuló las que a su juicio son las diez grandes preguntas de investigación de las ciencias de la tierra:

1. ¿Cómo se formaron la Tierra y otros planetas?

2. ¿Qué sucedió durante la "edad oscura" de la Tierra (primeros 500 millones de años)?

3. ¿Cómo comenzó la vida?

4. ¿Cómo funciona el interior de la Tierra y cómo afecta esto su superficie?

5. ¿Por qué la Tierra tiene tectónica de placas y continentes?

6. ¿Cómo son controlados los procesos de la Tierra por las propiedades de sus materiales?

7. ¿Qué causa el cambio climático y qué tanto puede ahora cambiar?

8. ¿Cómo la vida ha dado forma a la Tierra y cómo la Tierra ha moldeado la vida?

9. ¿Pueden predecirse los terremotos, las erupciones volcánicas y sus consecuencias?

10. ¿Cómo el flujo y transporte de fluidos afecta el ambiente antrópico? [38]

Previamente, en 1973, la Unesco y la Unión Internacional de Ciencias Geológicas (UICG) estructuraron el Programa Internacional de Geociencias (PICG), al que en 2015 se le adicionó el Programa de Geoparques Mundiales de la Unesco, para constituir el ahora denominado Programa Internacional de Geociencias y Geoparques (IGGP), Este programa se ha enfocado en el apoyo a la investigación y el intercambio científico en los siguientes cinco temas estratégicos:

\) 〉 del agua

〉) dad
Recursos de la Tierra: mantenimiento de nuestra socie-

Cambio global: evidencia de los registros geológicos

Riesgos geológicos: mitigar los riesgos

Hidrogeología: ciencias de la tierra inherentes al ciclo

Geodinámica: control de nuestro entorno 


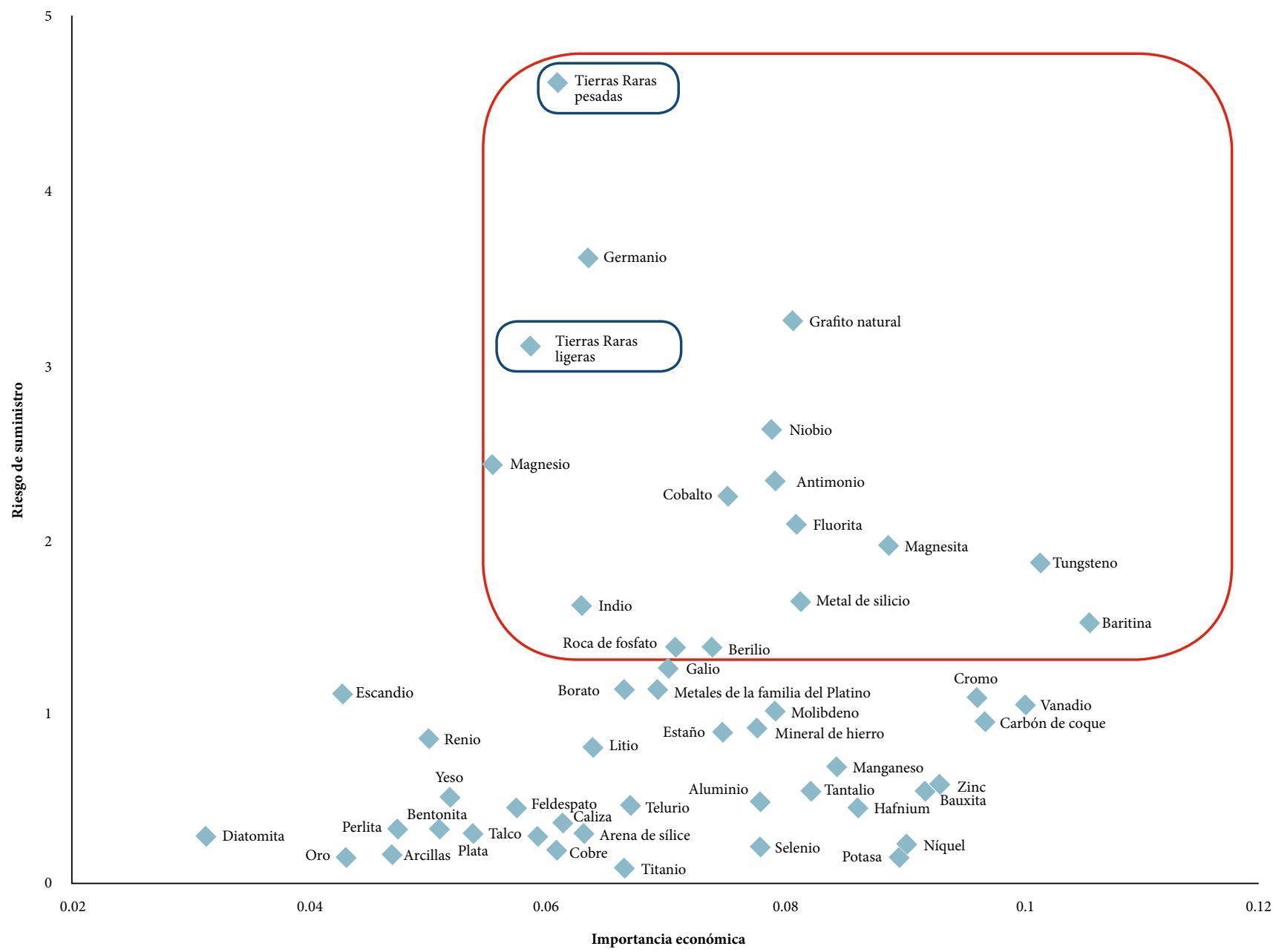

Figura 4. Minerales de importancia crítica en la OCDE, 2015 [14]

Otro tema de singular importancia en el ámbito geocientífico prospectivo se relaciona con la provisión de los recursos minerales, materias que fundamentan su valor en la utilidad que prestan y en la subsecuente abundancia/escasez de estos en la naturaleza. En el documento "Critical minerals today and in 2030: An analysis for OECD countries (2015)" [14] se muestra que las materias primas son esenciales para la economía global y que el desarrollo futuro depende de su suministro continuo, al igual que el de los combustibles fósiles, materiales que no son renovables. En general, sus depósitos en la corteza terrestre se encuentran agrupados o concentrados en zonas de similares características geológicas, lo que hace que la seguridad del suministro sea un riesgo potencial. En muchos casos el agotamiento de los depósitos minerales económicamente competitivos en los países industrializados ha hecho que los suministros dependan cada vez más de la estabilidad política de las economías emergentes ricas en minerales. De esta manera, la demanda creciente de estos mer- cados emergentes, nuevas tecnologías que requieren grandes cantidades de los minerales, la baja capacidad de sustitución en aplicaciones y las bajas tasas de reciclaje han incidido en que estas economías sean cada vez más vulnerables a posibles interrupciones en el suministro dirigido a los mercados globales.

En la figura 4 se muestra un estimativo combinado del riesgo relativo de suministro de minerales con respecto a su importancia económica para los países de la Organización para la Cooperación y el Desarrollo Económicos (OCDE), para 2015. Los minerales más representativos (es decir, los diez primeros) son, en orden descendente, baritas, tungsteno, vanadio, carbón de coque, cromo, zinc, níquel, potásicos y magnesita; sin embargo, esta última es la única que se encuentra entre los diez primeros en riesgo de suministro [14]. En esta misma línea argumentativa, el proyecto EU-Intraw (acrónimo de European Union's International Observatory for Raw Material) ha visualizado, mediante la metodología 
prospectiva apoyada de forma conceptual por el programa Micmac $^{\circledast}$ (acrónimo de matrice d'impacts croisés-multiplication appliquée à un classement [matriz de impactos cruzados y multiplicación aplicada para una clasificación]), tres probables escenarios de suministro de materias primas minerales para la Unión Europea en 2050 [15]. Este proyecto, construido con una visión de largo plazo, explora el consumo de estos geomateriales bajo el supuesto de que las políticas, estrategias y prioridades se encuentran en constante estado de flujo, en respuesta a los cambios políticos y socioeconómicos. Esta interdependencia configura una de las principales dimensiones del ya complejo sistema de suministro de materias primas minerales. El primer escenario, "Alianza para la sostenibilidad", examina la dinámica de un ambiente político y económico global que se centra en la sostenibilidad ambiental y económica, y que conduce cada vez más hacia una economía circular de materias primas. El segundo escenario, de "Comercio Ilimitado", refleja un mundo en el cual el libre comercio continúa dominando la política mundial y el entorno económico, con expectativas de una creciente demanda de materias primas. Finaliza con un tercer escenario, de los "Muros nacionales", que refleja un futuro en el que el nacionalismo y el proteccionismo económico comienzan a dominar, lo que conduce al estancamiento del crecimiento económico y a la volatilidad de las materias primas (commodities transables en los mercados de valores) [15].

Igualmente álgidos son los temas que, incorporados en la reflexión prospectiva, se relacionan con la emergente disciplina de la geoética, que

... consiste en la investigación y la reflexión sobre los valores que sustentan los comportamientos y las prácticas adecuadas, dondequiera que las actividades humanas interactúan con el sistema terrestre. La geoética se ocupa de las implicaciones éticas, sociales y culturales de la educación, la investigación y la práctica en geociencias, y del papel social y la responsabilidad de los geocientíficos en la realización de sus actividades [...] considerando los futuros del moderno desarrollo social y económico [16].

El geólogo Antonio Stoppani (1824-1891) introdujo el concepto de era antropozoica. Identificó a los humanos como una nueva "fuerza geológica" y, por lo tanto, como una parte integral y esencial de la naturaleza. Como primer populari- zador del conocimiento geológico, Stoppani se convirtió en uno de los pioneros de la geoética. Esta reconoce la contingencia de la evolución humana en el planeta e identifica al Homo sapiens como una fuerza geológica que actúa en los entornos geológico y biológico, y asigna a los humanos una responsabilidad ética que surge de la conciencia de ser un modificador del sistema terrestre [16]. La geoética nació en 1991 del cruce de la ética con la geología. El Dr. Vaclav Nemec es considerado el padre de esta disciplina. Tanto las ciencias de la Tierra como las Sociales han aceptado la geoética debido a la necesidad de una actitud ética apropiada para toda la geosfera y un análisis crítico de los dilemas geoéticos y la búsqueda de formas de resolverlos, que en todo caso deben asegurar la sostenibilidad de las generaciones futuras [16].

En este escenario de la geoética, bien vale la pena contextualizar como ejemplo algunos de los desarrollos de la Convención de las Naciones Unidas de Lucha contra la Desertificación (UNCCD), mediante el informe "Perspectiva global de la Tierra" [17]. Este estudio presenta una visión transformadora de la forma en que usamos la Tierra, factor determinante de nuestro bienestar futuro e hilo conductor de la estructura social de la humanidad. Se muestra un panorama mundial de la cartografía de la dinámica de la productividad de la Tierra, enfocado en la detección temprana de las trayectorias críticas de las transformaciones mundiales de la Tierra, observadas como el más importante desafío colectivo, "desde las presiones del crecimiento demográfico, el cambio climático, la urbanización, la migración y los conflictos a la inseguridad alimentaria, energética e hídrica. En todas sus dimensiones, la seguridad humana es cada vez más frágil y en muchas partes del mundo, la degradación de la Tierra y el cambio climático ya se reconocen como factores que contribuyen a una sensación de creciente inestabilidad”, asegura Monique Barbut, actual secretaria ejecutiva de la Convención de las Naciones Unidas de Lucha contra la Desertificación [17]. En la figura 5 se exhibe un esquema de las principales variables en las que se fundamenta la "seguridad humana", entendida como "el acceso a alimentos y agua, estabilidad de trabajo y medios de subsistencia, resiliencia ante el cambio climático y los fenómenos climáticos extremos y, en última instancia, estabilidad social y política”. Como conclusión de estudio es posible encontrar un futuro en el que exista una seguridad humana basada en la gestión de la Tierra, si se adopta la neutralidad de la degradación de 


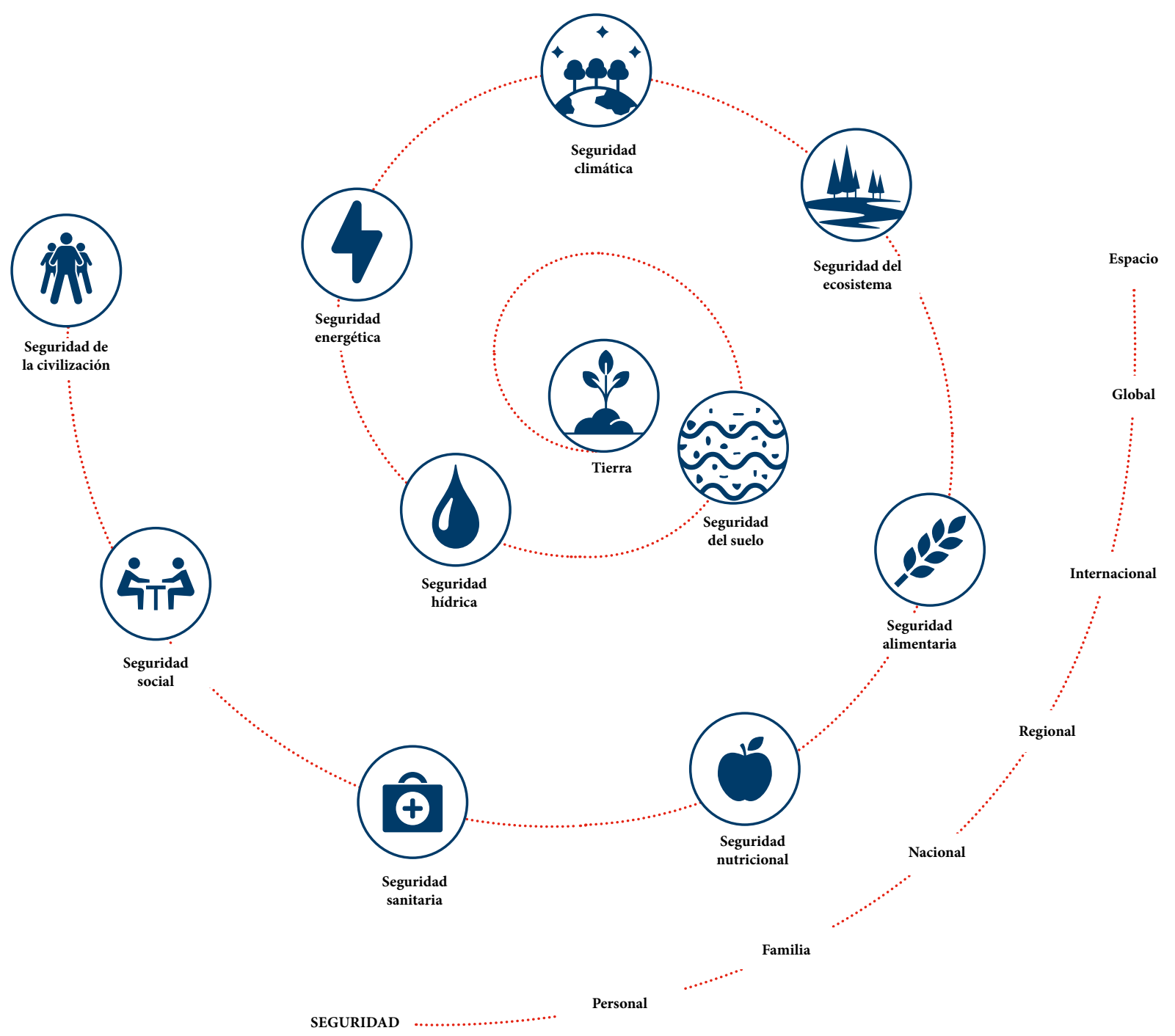

Figura 5. Dimensiones de la seguridad humana, 2017, UNCCD [17]

las tierras (NDT) como "un nuevo enfoque de la gestión de la Tierra que pretende fomentar acciones para evitar o reducir la degradación, y también para restaurar las tierras degradadas, con el fin de lograr el objetivo de pérdida neta cero en tierras sanas y productivas, a nivel nacional" [17].

Estas breves anotaciones tan solo pretenden llamar a la reflexión colectiva sobre la importancia de las geociencias integradas en los desarrollos conceptuales de la disciplina prospectiva, donde uno de los más recientes retos se relaciona con el entendimiento de las escalas espacio-temporales geológicas en la geosfera, en particular, en el nivel urbano o urbanosfera, compuesta a su vez por la biourbanosfera, la atmourbanosfera, la hidrourbanosfera y la litourbanosfera, y cómo estas afectarán la permanencia de la especie humana en el planeta [18] [19] y [20].

\subsection{Prospectiva en ciencias nucleares}

El suministro de energía eléctrica a partir de reactores nucleares de potencia (nucleoelectricidad) es uno de los componentes de la seguridad energética, concepto integrado como una de las dimensiones de la seguridad humana, explicada en el numeral anterior [17]. La seguridad energética, dada su naturaleza multidimensional, no cuenta con una definición global que la cubra. Este término apareció en los albores del siglo XX, en un ambiente geopolítico de industrialización caracterizado por la emergencia de nuevas naciones y sus consecuentes problemas de defensa y soberanía, en los que sin duda el rol del suministro de los combustibles para las fuerzas armadas cobró importancia estratégica. Varias décadas después, en el ámbito civil, el suministro de petróleo para facilitar la movilidad de las personas, los negocios y mercancías, se convirtió en la im- 
pronta de una época de vertiginoso crecimiento económico, en la que los derivados del petróleo ya ocupaban el primer lugar en el consumo energético de las naciones [21]. Se destacan las siguientes definiciones relacionadas en la referencia citada:

\) La seguridad energética es la disponibilidad ininterrumpida de fuentes de energía a un precio asequible (Energy International Agency, 2017).

» La seguridad energética es la capacidad de evitar el impacto adverso de cortes del suministro de energía causados por eventos naturales, accidentales o intencionales que afectan los sistemas de suministro y distribución de energía y servicios públicos (Departamento de Defensa de los Estados Unidos, 2009).

» La inseguridad energética es la pérdida de bienestar económico que puede ocurrir como resultado de un cambio en el precio o la disponibilidad de energía (Bohi y Toman, 1996).

» La seguridad energética es la capacidad de una economía para garantizar la disponibilidad de energéticos de manera sostenible y oportuna, con precios que no afecten negativamente el desempeño económico (APERC, 2007).

» La seguridad energética es la prestación ininterrumpida de servicios energéticos vitales (IIASA, 2012).

» La seguridad energética es la capacidad de un país para satisfacer la demanda nacional de energía con suficiencia, oportunidad, sustentabilidad y precios adecuados, en el presente y hacia un futuro, que suele medirse por lustros y decenios más que por años (Navarrete, 2008).

» La seguridad energética es simplemente la baja vulnerabilidad de los sistemas energéticos vitales (Cherp y Jewwell, 2014).

1) La seguridad energética nacional se concibe como la acción del Estado orientada a garantizar el suministro de energía de manera sostenible medioambiental y económicamente, a través del abastecimiento exterior y la generación de fuentes autóctonas, en el marco de los compromisos internacionales. La Estrategia de Seguridad Nacional plantea como objetivo en el ámbito de la seguridad energética diversificar las fuentes de energía, garantizar la seguridad del transporte y abastecimiento e impulsar la sostenibi- lidad energética. Entre las líneas de acción estratégica que plantea para alcanzar este objetivo se centran en tres aspectos: el abastecimiento, la distribución y el consumo (Departamento de Seguridad Nacional, Gobierno de España, 2019).

En este escenario, la emergencia de las tecnologías nucleares provistas por los sectores militares en la Segunda Guerra Mundial cobró singular importancia estratégica en la definición de este conflicto y en sus posteriores desarrollos geopolíticos enmarcados en la Guerra Fría. A partir de entonces fueron notorios los avances en tecnologías nucleares para aplicaciones en los sectores industrial, biomédico, bélico, ciencias básicas, ambientales y nanotecnologías, entre otras, y en la investigación teórica asociada a la modelación de las reacciones químicas nucleares.

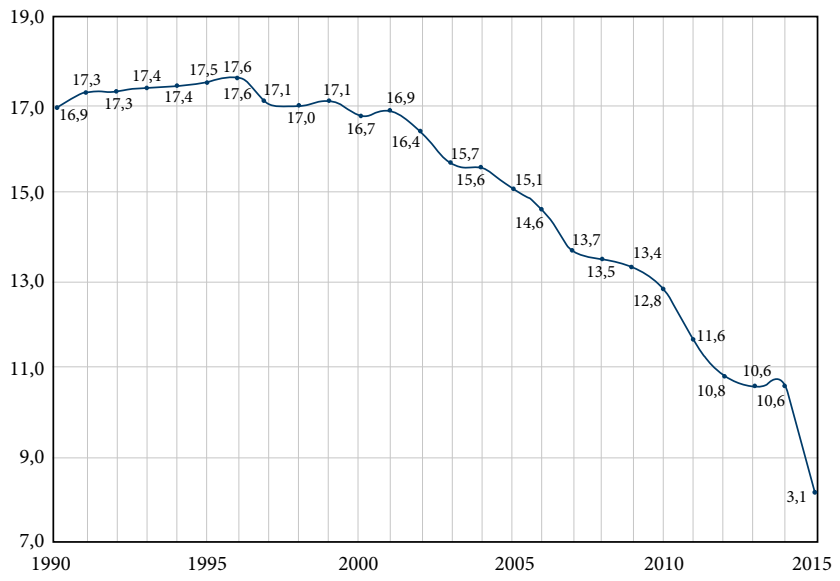

Figura 6. Producción histórica de electricidad a partir de fuentes nucleares [22]

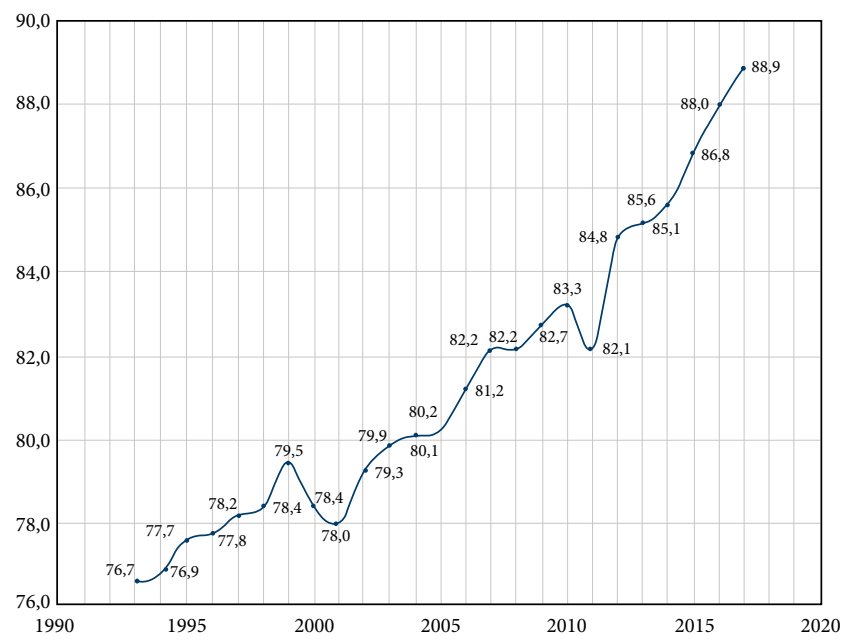

Figura 7. Acceso de la población mundial a la electricidad [22] 
Sin embargo, las recurrentes crisis petroleras $(1973,1979$ y 1990) empezaron a incidir también en la variación anual mundial del porcentaje de participación de la energía nuclear en la producción total de energía (figura 6). Allí se observa que si bien entre 1990 y 2015 esta se redujo a casi la mitad, actualmente algunos países, como Francia (77,6\%), Estados Unidos (19,3\%), Federación Rusa (17,0\%), Alemania (14,3\%), Bélgica (37,5\%), España (20,6\%), Reino Unido (20,9\%), Suecia $(34,7)$, Suiza $(34,9 \%)$ y Ucrania $(48,6 \%)$, presentan una dependencia estratégica significativa de esta fuente [22], que sopesada junto al acceso de la población mundial a la demanda de energía (figura 7), ofrece un interesante escenario para realizar elaboraciones de mayor complejidad.

Así, a escala mundial se aprecia un espectro promedio de generación de energía eléctrica (figura 8) que está ampliamente dominado por los combustibles fósiles (carbón, gas y petróleo, 66,7\%), mientras que la hidroelectricidad cubre el $17,2 \%$ y la nuclear $11,2 \%$. Las otras fuentes alternativas de la canasta energética son las provenientes de biomasa, energía eólica, geotérmica y solar.

Con respecto al consumo mundial final de energía en 2017 (figura 9), según datos de la OCDE, la Agencia Internacional de Energía y el Balance Prospectivo Nacional de Energía de México (2017), hubo un predominio de los sectores de industria y transporte, seguidos del uso residencial.

La energía nuclear, según lo confirmado por el reciente Panel Intergubernamental de las Naciones Unidas sobre el Cambio Climático (IPCC), en su informe emitido en octubre del 2018, podría desempeñar un importante papel en la transición energética necesaria orientada hacia un escenario mixto en el que primen las bajas emisiones de carbono [25].

Sin embargo, no se debe olvidar que la capacidad total de generación nuclear ha disminuido en casi un 10\% desde 2006, lo cual resulta, en la actualidad, en una participación mundial en la generación de electricidad de cerca de, tan solo, el $11 \%$. Esto se debe principalmente al cierre temporal de varias plantas nucleares en Japón después del evento de Fukushima, seguido de otros cierres permanentes en este mismo país, los Estados Unidos y Europa. En algunos casos, este descenso es el resultado de una decisión política de eliminación/reducción ("moratoria") de la opción nuclear; en otros, de la falta de competitividad de centrales nucleares en los mercados de electricidad en evolución, atribuida a los bajos precios del gas natural o la emergencia de las energías renovables subsidiadas.

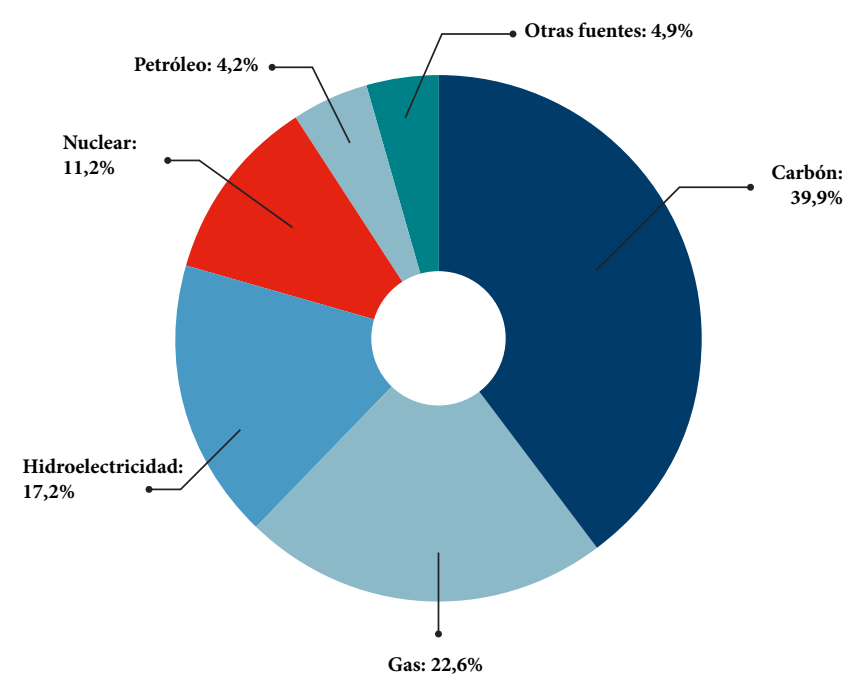

Figura 8. Panorama mundial de generación eléctrica según fuentes de energía [23]

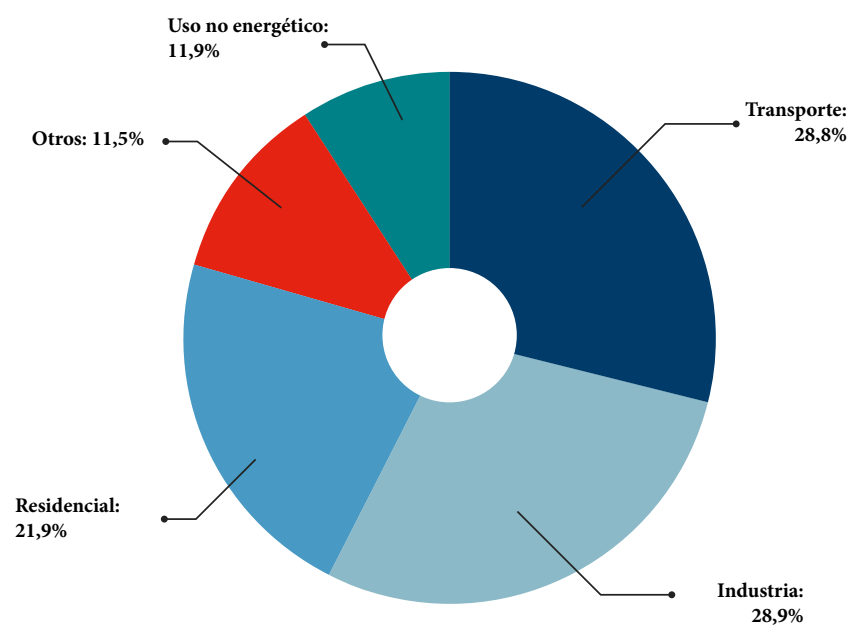

Figura 9. Consumo mundial final de energía por sectores, 2018 [24]

Sobre este aspecto, vale resaltar que, aunque se están construyendo varios reactores nucleares de potencia, principalmente en la República Popular China y en la Federación Rusa, la mayoría de los proyectos de nueva construcción en los Estados Unidos de América y en la región de Europa occidental ha sufrido retrasos en sus cronogramas y sobrecostos en sus presupuestos, lo que ha hecho que los posibles inversionistas se muestren reacios a asumir compromisos mayores que los requeridos con antelación. A esto se suma la creciente pérdida de credibilidad, entre la opinión pública informada, de la industria nuclear, que ha derivado en un impacto mediático negativo por la oposición de ONG ambientalistas y partidos políticos verdes, entre otros actores de la sociedad civil organizada, por los recientes incidentes en centrales nucleoeléctricas [26]. 
Generación I

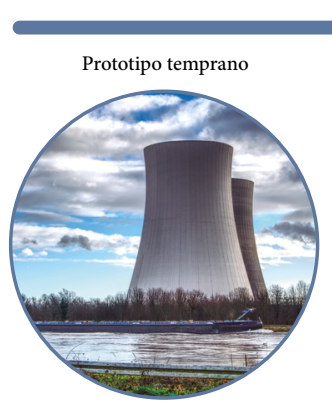

Generación II

Generación III

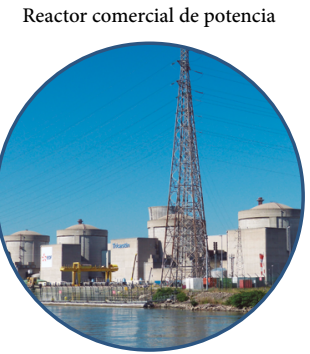

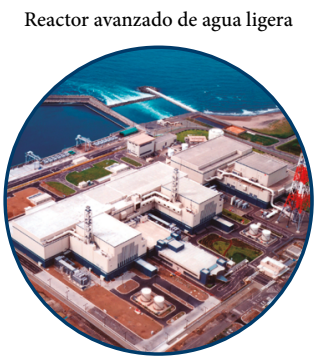

Generación III+

Generación IV
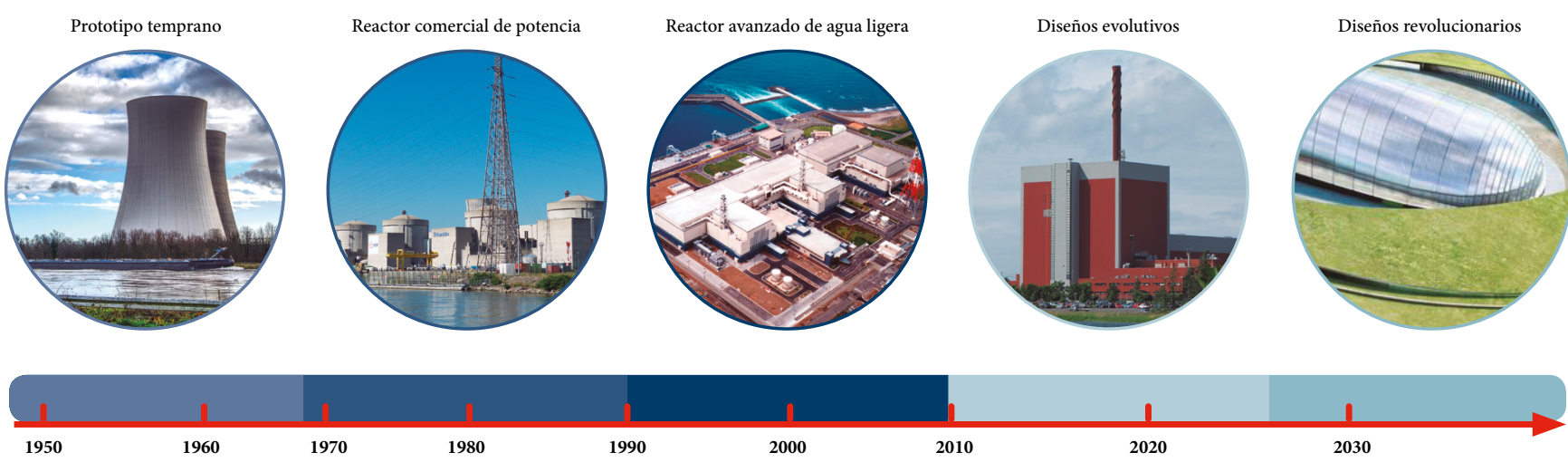

Figura 10. Generaciones de los reactores nucleares de potencia (NPP, por su acrónimo inglés) [27]

Este panorama un tanto sombrío no ha sido óbice para que la industria nuclear continúe sus desarrollos tecnológicos. En la figura 10 se muestra un esquema de la línea de tiempo del desarrollo de los reactores nucleares de potencia (nuclear power plants, NPP), incluyendo las previsiones a 2030, postulado por el Foro Internacional de Reactores de Generación IV (GIF, por su acrónimo inglés). Estos desarrollos tecnológicos se fundamentan en cuatro principios básicos: sostenibilidad; seguridad y credibilidad; competitividad económica y protección física [27].

Los reactores de potencia de cuarta generación (proyectados como factibilidad) se caracterizan por su alta eficiencia en el diseño, que garantizará la posibilidad de generar combustible fisible a partir de material fértil, con lo cual se produciría un material igual o más fisible de lo que consume el reactor en condiciones normales de operación.

La implantación de estas tecnologías se traduciría en una significativa reducción de los pasivos ambientales ocasionados por la construcción de repositorios geológicos profundos que usualmente albergan los desechos producidos en estas instalaciones nucleares, mientras se desarrollan tecnologías que aumenten la eficiencia en el reprocesamiento de combustibles usados. La emergencia de las actuales tecnologías fue prevista de manera acertada en el ejercicio prospectivo de 2002 realizado por el mencionado foro, en el que, de cien ideas conceptuales, fueron seleccionadas como promisorias las siguientes:

» Reactor rápido enfriado con plomo (LFR)

» Reactor de sal fundida (MSR)
\) Reactor rápido refrigerado por sodio (SFR)

\) Reactor supercrítico refrigerado por agua (SCWR)

» Reactor de muy alta temperatura (VHTR)

De este escenario también suelen mencionarse como ideas disruptivas los denominados pequeños reactores modulares (small modular reactors, SMR), que, en términos del OIEA se definen como una "nueva generación de reactores nucleares diseñados para generar energía eléctrica de hasta $300 \mathrm{MW}$, cuyos componentes y sistemas pueden fabricarse en una instalación externa y luego ser transportados como módulos a los sitios donde la demanda implique su instalación" [28].

Para países como el nuestro, con una matriz energética poco diversificada y escaso o ningún desarrollo de generación eléctrica mediante reactores nucleares de potencia previsto en su planeación, alternativas como la mostrada con los SMR pueden coadyuvar en el eventual suministro seguro de energía en vastas zonas no interconectadas (ZNI) de la compleja geografía nacional.

Actualmente, en un reciente boletín (junio de 2019) de la Asociación Nuclear Mundial (World Nuclear Association, WNA), se presentó un panorama medianamente promisorio de generación nucleoeléctrica en los países emergentes, es decir, aquellos que están considerando, planean o han empezado la construcción de reactores nucleares de potencia. Entre ellos se destacan los siguientes:

») En Europa: Italia, Albania, Serbia, Croacia, Portugal, Noruega, Polonia, Bielorrusia, Estonia, Letonia, Lituania, Irlanda y Turquía. 
Tabla 6. Reactores nucleares de potencia operativos en América [40]

\begin{tabular}{cccc}
\hline País & N. ${ }^{\circ}$ de reactores operativos & N. de reactores en construcción & \% total en matriz energética \\
\hline EE.UU. & 99 & 5 & $19,4 \%$ \\
\hline Canadá & 19 & 0 & \\
\hline México & 2 & 0 & \\
\hline Brasil & 2 & 1 & \\
\hline Argentina & 3 & 1 & \\
\hline TOTAL & 125 & $4,0 \%$ & \\
\hline
\end{tabular}

En el Medio Oriente y África del norte: estados del golfo, incluidos los Emiratos Árabes Unidos, Arabia Saudita, Qatar y Kuwait; Yemen, Israel, Siria, Jordania, Egipto, Túnez, Libia, Argelia, Marruecos y Sudán.

En África occidental, central y meridional: Nigeria, Ghana, Senegal, Kenia, Uganda, Tanzania, Zambia, Namibia y Ruanda.

〉> nezuela, Bolivia, Perú y Paraguay.

» En Asia central y meridional: Azerbaiyán, Georgia, Kazajstán, Mongolia, Bangladesh, Sri Lanka y Uzbekistán.

» En el sudeste de Asia y Oceanía: Indonesia, Filipinas, Vietnam, Tailandia, Laos, Camboya, Malasia, Singapur, Myanmar, Australia y Nueva Zelanda.

En el este de Asia: Corea del Norte.

La situación de los reactores nucleares de potencia en 2015 se muestra en la tabla 6. En ese entonces se destacaba su participación en la matriz energética de los Estados Unidos, que en 2019 se mantuvo en 19\%, con un incremento de la participación del gas natural (37\%) y las energías renovables (19\%), previéndose la expansión de esta últimas a 38\% hacia 2050. A pesar del número significativo de naciones (cerca de 30) involucradas en la generación nucleoeléctrica, no se prevé que estos desarrollos contribuyan significativamente a la expansión y consolidación de una capacidad nuclear "deseable" en un futuro próximo [29].

Como conjunto, la situación energética en el entorno latinoamericano no se diferencia mucho de la mundial. Áreas geográficas con abundantes recursos naturales (combustibles fósiles y biomasa) y una climatología que favorece la producción hidroenergética son las características regionales relevantes [30]. Esta situación predetermina que de manera lenta la matriz de oferta energética (2016) se diversifique, dominada ampliamente por el consumo de petróleo y sus derivados (39\%), seguido del gas natural (30\%) y el carbón (6\%). Posteriormente, la hidroelectricidad cubre el 7\%, mientras la energía nuclear apenas alcanza el $1 \%$. Se destaca cómo la biomasa llega a 16\%, y las otras energías renovables, al $1 \%$.

La oferta diversificada de la actual matriz energética (2016) en la región de América Latina y del Caribe se muestra en las gráficas 11 y 12 , resultado de una detallada simulación desarrollada por los investigadores en prospectiva energética de la Organización Latinoamericana de Energía (Olade) y orientada hacia el escenario de 2040, para cuando se prevé una progresiva inclusión de otras energías renovables (solar, eólica y geotérmica, entre otras) que, en todo caso, superan la expectativa de avance de la introducción de la energía nuclear. Esta última mantendrá, según este estudio, una participación marginal en el concierto energético regional cercana al $1 \%$.

En el contexto histórico latinoamericano fueron pioneros en los desarrollos conceptuales de los programas nucleares, Brasil, en la década de los treinta del siglo XX, durante el gobierno de Getulio Vargas, Argentina en los cuarenta, y México, en los años cincuenta, esfuerzos que se concretaron en 1974 con la puesta en funcionamiento de Atucha I, la primera central nucleoeléctrica de la región (Argentina). Actualmente, cada uno de estos países posee dos centrales de generación nucleoeléctrica operativas [32]. En el ámbito local, en Colombia el primer intento por introducir en la planeación energética indicativa la variable nuclear se dio en la década de los ochenta, en la Administración Betancur (1982-1986), cuando se publicó el documento "Demanda de energía en el estudio nacional de energía (ENE), de Colombia: metodología y resultados", en cuyo marco conceptual se formuló el primer Plan de Desarrollo Nuclear (PDN), documento estratégico en el que se plantearon las tres etapas estratégicas necesarias para la consolidación de esta tecnología en nuestra nación [33]:

Etapa I, 1985-2002. Optimización y conversión del reactor nuclear IAN-R1, orientada hacia la utilización de un combustible de bajo enriquecimiento ( $\mathrm{LEU} 20 \% \mathrm{U}_{3} \mathrm{O}_{8}$ ) y la elevación de la potencia hasta un megavatio $(1.000 \mathrm{~kW})$, fomento 
de la producción de radioisótopos y la aplicación intensiva de las técnicas analíticas nucleares en diversos sectores productivos.

Etapa II, 2002-2022. Diseño y construcción de un centro nuclear de investigación y producción. Construcción y puesta a crítico de un nuevo reactor multipropósito, en las afueras de la ciudad, y evaluación integral de la prefactibilidad de la capacidad de generación nucleoeléctrica mediante modelos proyectivos.

Etapa III, 2022-2035. Primer reactor nuclear de potencia. Introducción del país en la era de la nucleoelectricidad.

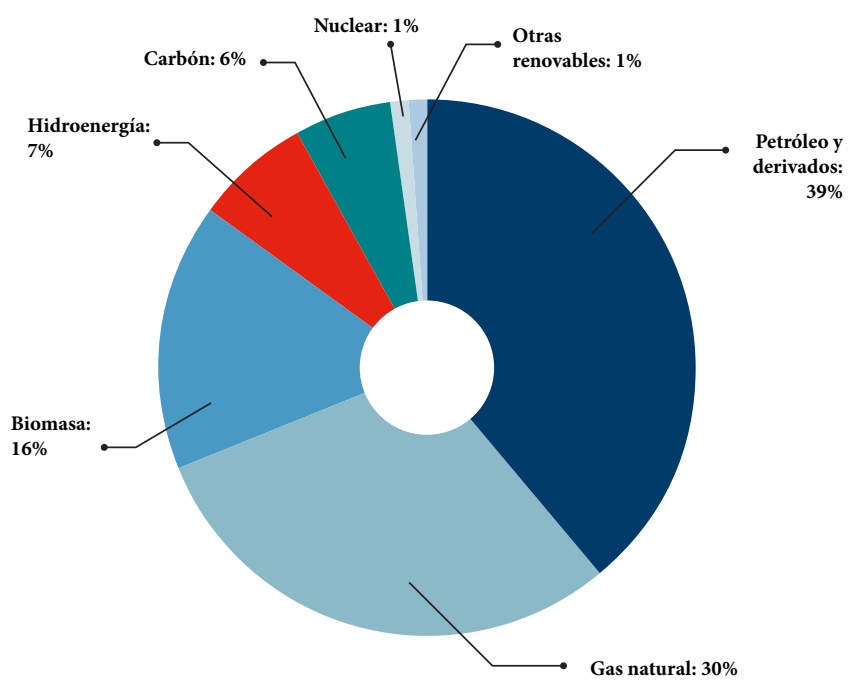

Figura 11. Matriz energética de América Latina y el Caribe en 2016, según la Olade [31]

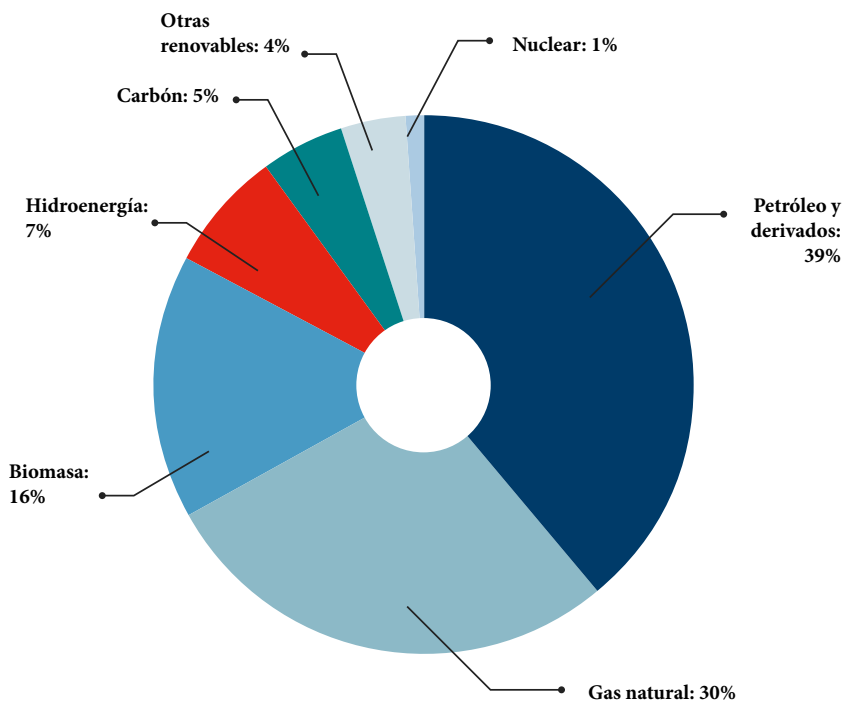

Figura 12. Simulación de la matriz energética de América Latina y el Caribe proyectada para 2040, según la Olade [31]
La evidencia existente a la fecha indica que este plan nunca se concretó, en parte por la falta de visión estratégica de futuro de nuestros planificadores y, por otro lado, debido a la abundancia de recursos hídricos de ese entonces, que pospusieron esta decisión. Posteriores eventos relacionados con las anomalías climáticas (fenómeno de El Niño) develaron esta falta de previsión y sometieron a los ciudadanos a intensos racionamientos de energía a principios de la décadas de los noventa del pasado siglo, cuyo fantasma - la "inseguridad energética" - volvió a aparecer, en concurrencia con la conocida situación del proyecto Hidroituango (que representaba el 17\% de la demanda de energía eléctrica nacional a finales de 2018, según fuentes acreditadas de la propia EPM/Medellín, Col.).

Con el objetivo de prever, reducir, mitigar y eventualmente compensar los efectos negativos — algunos de ellos poco previsibles - generados y no contemplados en los ejercicios formales de prospectiva, la Unidad de Planeación Minero-Energética (UPME), el Departamento Nacional de Planeación (DNP), el Ministerio de Minas y Energía, la Interconexión Eléctrica S. A. (ISA), las Empresas Públicas de Medellín (EPM) y algunos centros consultores del pensamiento prospectivo, entre otros, han avanzado durante las últimas décadas en los refinamientos para construir herramientas metodológicas relacionadas. En 2010 se presentó el "Plan de desarrollo para las fuentes no-convencionales de energía en Colombia” (PDFNCE), orientado a la evaluación de los recursos solar, eólico, de pequeñas centrales hidroeléctricas $(\mathrm{PCH})$ y biomasa, y se encontró que la información sobre los recursos geotérmico, nuclear y el aprovechamiento energético de los océanos era bastante preliminar, cuando no prácticamente inexistente. En particular, en las zonas no interconectadas (ZNI), la eventual dotación o potencial de estos recursos sigue siendo baja, en buena medida explicada por la escasa población que allí se asienta. Respecto a la energía nuclear, el estudio concluye que esta "se visualiza en el muy largo plazo, para lo cual se considera conveniente ir construyendo las condiciones que lo permitan cuando ello resulte conveniente para el país desde el punto de vista de seguridad energética y de costos" [34].

Un interesante ejercicio prospectivo, el "Plan energético nacional Colombia: Ideario energético 2050”, realizado por la UPME en 2015, establece que en un escenario de largo plazo (2014-2050) se privilegie en el país una transición de la canasta energética hacia las energías renovables, limpias, 
adaptadas al cambio climático y enfocadas en la entrega de servicios energéticos con mayor eficiencia.

En el mismo estudio se reflexiona acerca de resistencia generada por el eventual uso de las técnicas de fractura hidráulica (fracking), sobre la decisión de la política alemana de desmontar su parque nuclear y la posibilidad de establecer una línea de política relacionada con nuevos marcos regulatorios que favorezcan el desarrollo de la energía nuclear en el país. Esta alternativa estaría ligada con la construcción de pequeñas centrales nucleares (PCN), que

... pueden verse como una alternativa a largo plazo para diversificar el parque generador colombiano. La energía nuclear es una fuente confiable, segura y libre de emisiones de efecto invernadero. En Europa y Estados Unidos han venido trabajando en esquemas de negocios que permiten flexibilizar el uso de los combustibles para centrales nucleares y se ha desarrollado una opción de "nuclear fuel leasing" que permite al operador de una central poner el riesgo y costos asociado al combustible nuclear, incluyendo su disposición final, bajo la responsabilidad de un tercero. Esto minimiza los requisitos regulatorios previos a la instalación, que generalmente son muy engorrosos. Esta opción, combinada con las nuevas tecnologías de alto nivel de seguridad, como por ejemplo el "tristructural isotropic fuel", y los menores tamaños de las unidades (de $50 \mathrm{MW}$ o menos), vuelven a la energía nuclear una opción a considerar en el largo plazo. [35]

Un posterior estudio realizado por el Departamento Nacional de Planeación (DNP) en 2017, con el apoyo del Banco Mundial y el Fondo Fiduciario de Crecimiento Verde de Corea, para aportar en los insumos de "construcción de políticas públicas en el contexto de la Misión de Crecimiento Verde, liderada por el DNP", aborda los temas relacionados con la oferta de energía enfocándose en fuentes no convencionales de energía renovables (FNCER), entre las cuales la opción nuclear se encuentra incluida, de acuerdo con lo estipulado por la Ley 1715 de 2014, que regula la integración de las energías renovables no convencionales al Sistema Energético Nacional, y que menciona en su

Artículo 5. ${ }^{\circ}$ Definiciones. Numeral 16. Fuentes no convencionales de energía (FNCE). Son aquellos recursos de energía disponibles a nivel mundial que son ambiental- mente sostenibles, pero que en el país no son empleados o son utilizados de manera marginal y no se comercializan ampliamente. Se consideran FNCE la energía nuclear o atómica y las FNCER. Otras fuentes podrán ser consideradas como FNCE según lo determine la UPME. [36]

Entre los desarrollos del mencionado estudio se encuentra la alianza estratégica constituida por la UPME, la Universidad del Valle, Instituto de Prospectiva, la Universidad Nacional de Colombia, Grupo de Investigación del Sector Energético Colombiano (Grisec) y la Corporación Centro de Desarrollo Tecnológico del Gas, que a mediados del 2018 emitió el informe de propuesta para la conformación del Observatorio Colombiano de Energía (OCE), espacio de reflexión sobre el futuro energético de nuestra nación con una perspectiva a 2050 [37]. En ese informe, dada su naturaleza prospectiva, se identifican como viables las siguientes tendencias de alto impacto:

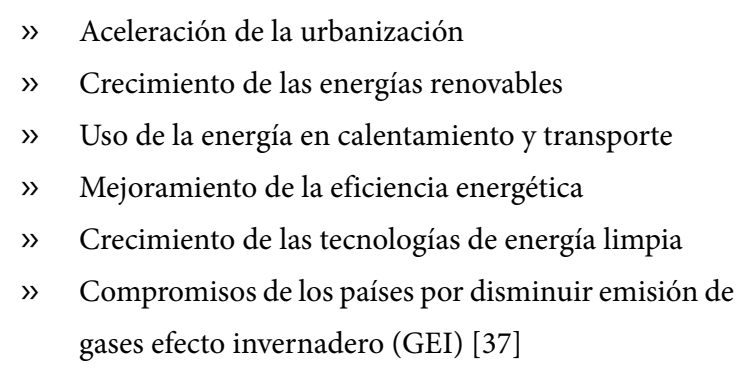

Asimismo, se consideran estas tendencias de bajo impacto:
Generación de electricidad a partir de energía nuclear In- cremento de la demanda de GLP (gas licuado de petróleo) para hogares y zonas rurales [37]

La prospectiva energética, como elemento clave de la "seguridad energética", es una de las temáticas estratégicas en que el SGC se debe involucrar con otros actores (UPME, ISA, MinEnergía, Isagén, Acolgén, DNP, grupos de investigación de MinCiencias, OIEA, OCDE, IEA, centros académicos y del pensamiento, observatorios, etc.), para contribuir desde sus competencias al avance del conocimiento, insumo básico para la generación de políticas públicas de largo plazo que articulen la generación nucleoeléctrica con los planes de diversificación de la canasta energética nacional. 


\section{CONCLUSIONES}

El abordaje de los estudios prospectivos o de futuro en el Servicio Geológico Colombiano, en sus componentes geocientífica y nuclear, es una tarea pendiente de realizar, tanto por sus investigadores como por el estamento directivo de la entidad. Siendo notable la producción conceptual y práctica sobre la disciplina prospectiva, disponible en los diferentes repositorios virtuales de información, es necesario que la entidad forme y capacite a funcionarios y colaboradores en el manejo de las herramientas informáticas de la prospectiva y en las disponibles de forma gratuita en la web, por ejemplo, en http://es.laprospective.fr/Metodos-de-prospectiva/ Los-programas.html.

Estos breves apuntes de prospectiva pretenden favorecer una cultura de la indagación, la autocrítica y el debate mediante la exposición y confrontación de ideas y argumentos técnicos, elementos básicos de los ejercicios prospectivos o de construcción de futuro, donde la elaboración de planes estratégicos con horizontes de tiempo de diez, veinte y treinta años se convierta en una buena práctica de gerencia pública que ilumine el desarrollo institucional a largo plazo.

Esto se traduce en el ejercicio de repensar la actual institucionalidad pública de ciencia y tecnología al amparo de los futuros deseables que se pretenden establecer para ella, visualizando como efecto neto la mejora objetiva de la calidad de vida de la población colombiana.

\section{Agradecimientos}

El autor agradece de forma especial a los integrantes de la Dirección Técnica de Asuntos Nucleares (DTAN) del Servicio Geológico Colombiano, quienes aportaron ideas que fortalecieron las bases conceptuales de construcción de este escrito.

\section{Referencias}

[1] Sun Tzu, El arte de la guerra. 2015. FeedBooks/Electronic-book. Disponible en http://cimcon.armada.mil. co/sites/default/files/El\%20arte\%20de\%20la\%20guerra.\%20Sun\%20Tzu.pdf

[2] G. Walta, "La literatura de anticipación". Revista Telos, Fundación Telefónica n. ${ }^{\circ}$ 108. [En línea]. Disponible en https://telos.fundaciontelefonica.com/wp-content/uploads/2017/11/telos-108-analisis-gabriel-h-walta.pdf
[3] J. Medina, S. Becerra y P. Castaño, Prospectiva y política pública para el cambio estructural en América Latina y el Caribe. Libros de la Cepal, n. ${ }^{\circ} 129$ (LC/G.2622-P). Santiago de Chile: Comisión Económica para América Latina y el Caribe, 2014.

[4] C. Mera, "Concepto, aplicación y modelo de prospectiva estratégica en la administración de las organizaciones". Revista Estrategia Organizacional, UNAD. [En línea]. Disponible en http://academia.unad.edu.co/images/investigacion/hemeroteca/estrategiaorganizacional/2012/ Concepto,\%20aplicaci\%C3\%B3n\%20y\%20modelo.pdf

[5] M. Bunge, La investigación científica, Barcelona: Siglo XXI Editores. 2004.

[6] E. Morin, Introducción al pensamiento complejo. Barcelona: Gedisa. 2001.

[7] E. Morin, Los siete saberes necesarios para la educación del futuro. Bogotá: Cooperativa Editorial Magisterio. 2001.

[8] A. Afuah, La dinámica de la innovación organizacional. México, D. F.: Oxford University Press. 1999.

[9] M. Godet, De la anticipación a la acción. Barcelona: Marcombo. 1993.

[10]E. Ortegón y J. Medina, Prospectiva: construcción social del futuro. Cali: Ilpes y Universidad del Valle. 1997.

[11] S. Tooth, M. J. Smith, H. A. Viles y F. Parrott. Perspectives on the contemporary art-geoscience interface. Special volume, Jornal of Maps., vol. 15: 3. 2019. Disponible en https://www.tandfonline.com/toc/tjom20/15/3?nav=tocList

[12] G. Baena, G. "Sobre futuros incompletos y esperanzas continuas... líneas para la historia de la prospectiva en México". Selected Readings: Foresight experiences in European Union \& Latin America, under the $7^{\text {th }}$ Framework Programme for Research and Technological Development Workshop. Flacso-México. 2008.

[13]D. Galloway, “The complex future of hydrogeology”. Hydrogeology Journal, 18, pp. 807-810. 2010. Disponible en https://www.researchgate.net/profile/Devin_Galloway/ publication/225443668_The_complex_future_of_Hydrogeology/links/556f037008aefcb861dd4a87.pdf

[14] OCDE, "Critical minerals today and in 2030: An analysis for OECD countries". París. 2015.

[15] S. Schimpf, F. Sturm, V. Correa, B. Bodo y C. Kean, “The world of raw materials 2050: Scoping future dynamics in 
raw materials through scenarios". Energy Procedia, vol. 125, pp. 6-13. 2017.

[16] P. Bobrowsky, V. S. Cronin, G. di Capua, S. W. Kieffer y S. Peppoloni, "The emerging field of geoethics". En Scientific Integrity and Ethics with Applications to the Geosciences, Special Publication American Geophysical Union, John Wiley and Sons, Inc. 2017.

[17] Naciones Unidas, "Convención de Lucha contra la Desertificación. Informe de perspectiva global de la tierra”. $1^{\text {a }}$. ed. Bonn. 2017. ISBN: 978-92-95110-52-6.

[18]R. Menegat, R. C. Fontana, "Gigantic cities and the new challenge of an old science: Geoethics, geoeducation, and geoknowledge in Porto Alegre, Brazil". En Geoethics in Latin America. The Latin American Studies Book Series. Springer International Publishing AG, part of Springer Nature. 2018. ISSN 2366-343X.

[19]R. L. Terrington, S. Thorpe, H. Kessler, A. Bidarmaghz, R. Choudhary, M. Yuan y S. Bricker, "Making geology relevant for infrastructure and planning". International Conference on Smart Infrastructure and Construction 2019 (ICSIC): Driving data-informed decision-making. Cambridge, UK: British Geological Survey, University of Cambridge. pp. 403-409. https://doi.org/10.1680/icsic.64669.403

[20]L. Cuervo y F. Guerrero, "Prospectiva en América Latina: aprendizajes para la práctica. Parte 2. Prospectiva urbana y local". Santiago de Chile: Cepal. Serie Seminarios y Conferencias. 2018. Disponible en https://repositorio. cepal.org/bitstream/handle/11362/43722/S1701160_es.pdf? sequence $=1$

[21]V. Rodríguez, "Seguridad energética: análisis y evaluación del caso de México". México D. F.: Cepal, serie Estudios y Perspectivas. 2018. ISSN 1680-8800.

[22] World Bank, Data Bank. [En línea]. Disponible en https://datos.bancomundial.org/indicator/EG.ELC. NUCL.ZS? end $=2015 \&$ start $=1990 \&$ view $=$ chart

[23] S. Buruchenko e I. Pioro, "Nuclear power as a basis for future electricity generation". World Multidisciplinary Earth Sciences Symposium (WMESSS). IOP Conference. 2017. https://doi.org/10.1088/1755-1315/95/4/042002

[24] Gobierno Federal de México, Informe del balance prospectivo energético nacional. 2018. [En línea]. https:// www.gob.mx/sener/documentos/prospectivas-del-sector-energetico. México D. F.
[25]B. W. Brook y J. A. C. Bradshaw, "Key role for nuclear energy in global biodiversity conservation". Conservation Biology, vol. 29, n. ${ }^{\circ}$ 3, pp. 702-712. 2014. https://doi. org/10.1111/cobi. 12433

[26] OCDE y NEA, Nuclear innovation 2050: An NEA initiative to accelerate $R \& D$ and market deployment of innovative nuclear fission technologies to contribute to a sustainable energy future. París. 2019

[27] OECD y NEA. Technology Roadmap Update for Generation IV Nuclear Energy Systems. París. 2014.

[28] International Atomic Energy Agency, Advances in small modular reactor technology developments: A supplement to IAEA dvanced Reactors Information System (ARIS). Vienna. 2016. [En línea]. Disponible en https://aris.iaea. org/Publications/SMR-Book_2016.pdf

[29]World Nuclear Association "Emerging nuclear energy countries: Report”. 2019. [En línea]. Disponible en https://www.world-nuclear.org/information-library/ country-profiles/others/emerging-nuclear-energy-countries.aspx

[30]F. Morales y A. Márquez, "Situación actual de la energía nuclear en América: proyecciones de una realidad asimétrica". Cuadernos de Difusión/Prospectiva y Estrategia: Visión de Futuro para las Políticas Públicas, n. ${ }^{\circ} 9$. Pontificia Universidad Católica de Chile. 2014.

[31] Organización Latinoamericana de Energía (Olade), Panorama energético de América Latina y el Caribe, 2018. Sistema de Información Energética de Latinoamérica y el Caribe (Sielac). Quito. 2018. [En línea]. Disponible en http://biblioteca.olade.org/opac-tmpl/Documentos/ old0416b.pdf

[32]R. Robayo, G. A. Sahid, J. E. Villareal y V. Manrique, 2014. Reflexiones del poder nuclear en el siglo XXI. Bogotá: Universidad del Rosario. ISSN 2027-615X n. ${ }^{\circ} 2$. 2014. Disponible en https://www.urosario.edu.co/ODA/ Archivos/doc_investigacion/Reflexiones-del-poder-nuclear-en-el-Siglo-XXI/

[33] G. Parrado, D. Alonso, M. Peña y F. Mosos, "Aplicaciones del reactor nuclear colombiano de investigación IAN-R1". Revista Anales de Ingeniería, n. ${ }^{\circ}$ 932, Sociedad Colombiana de Ingenieros. 2015. ISSN 0120-0149. Disponible en https://issuu.com/ingsci/docs/revista_932

[34] H. Rodríguez y F. González, Plan de desarrollo para las fuentes no-convencionales de energía en Colombia (PD- 
FNCE). Bogotá: Corpoema y UPME. 2010. Disponible en: https://bdigital.upme.gov.co/bitstream/001/994/1/ Vol\%201\%20Plan\%20Desarrollo.pdf

[35] Unidad de Planeación Minero-Energética, Plan Energético Nacional Colombia: Ideario energético 2050. Bogotá. 2015. Disponible en https://biblioteca.minminas. gov.co/pdf/Plan\%20energetico\%20Nacional\%202050. pdf

[36] Departamento Nacional de Planeación, World Bank Group, Misión de Crecimiento Verde, EnerSinc y Korea Green Growth Partnership, Energy demand situation in Colombia. 2017. [En línea]. Disponible en https://www. dnp.gov.co/Crecimiento-Verde/Documents/ejes-tematicos/Energia/MCV\%20-\%20Energy\%20Supply\%20 Situation\%20vf.pdf

[37] Unidad de Planeación Minero-Energética (UPME) y Universidad Nacional de Colombia, sede Bogotá, $\mathrm{Ob}$ - servatorio Colombiano de Energía: aproximación a las condiciones para su conformación. Informes de Vigilancia Tecnológica, Convenio 005-2017 UPME-UNAL. Bogotá. 2018.

[38] National Research Council. 2008. Origin and Evolution of Earth: Research Questions for a Changing Planet. Washington, DC: The National Academies Press. https://doi.org/10.17226/12161.

[39]Hauptman A., Steinmüller K. Surprising Scenarios. Imagination as a Dimension of Foresight. In: Peperhove R., Steinmüller K., Dienel HL. (eds) Envisioning Uncertain Futures. Zukunft und Forschung. Springer VS, Wiesbaden. https://doi.org/10.1007/978-3-658-250744_4. 2019

[40] International Atomic Energy Agency (2019). "Power Reactor Information System". [Internet]. Disponible en https://pris.iaea.org/PRIS/home.aspx 